\title{
A Hybrid Region Growing Algorithm FOR MEDICAL IMAGE SEgMENTATION
}

\author{
D. Muhammad Noorul Mubarak ${ }^{1}$, M. Mohamed Sathik ${ }^{2}$, S.Zulaikha Beevi ${ }^{3}$ and K. \\ Revathy $^{4}$ \\ ${ }^{1,4}$ Department of Computer Science, University of Kerala,Trivandrum, India. \\ noorul.muby@gmail.com revathy_srpeyahoo.com \\ ${ }^{2}$ Principal, Sathakathullah Appa College, Tirunelveli, Tamilnadu, India. \\ mmdsadiq@gmail . com \\ ${ }^{3}$ Department of IT, National College of Engineering, Tirunelveli, Tamilnadu, India. \\ zulaikha23@gmail.com
}

\begin{abstract}
In this paper, we have made improvements in region growing image segmentation. The First one is seeds select method, we use Harris corner detect theory to auto find growing seeds. Through this method, we can improve the segmentation speed. In this method, we use the Improved Harris corner detect theory for maintaining the distance vector between the seed pixel and maintain minimum distance between the seed pixels. The homogeneity criterion usually depends on image formation properties that are not known to the user. We induced a new uncertainty theory called Cloud Model Computing (CMC) to realize automatic and adaptive segmentation threshold selecting, which considers the uncertainty of image and extracts concepts from characteristics of the region to be segmented like human being. Next to region growing operation, we use canny edge detector to enhance the border of the regions. The method was tested for segmentation on $X$-rays, CT scan and MR images. We found the method works reliable on homogeneity and region characteristics. Furthermore, the method is simple but robust and it can extract objects and boundary smoothly.
\end{abstract}

\section{KEYWORDS}

region growing, segmentation, seeds selection, homogeneity criterion, cloud model

\section{INTRODUCTION}

The analysis of medical images such as X-rays, CT scan, MR images often requires segmentation prior to visualization or quantification and it is a challenging task. For segmentation of structures in CT images many different approaches exist [19] among which region growing is popular having the advantage of letting the user specify just one region that he/she is interested in [6]. Location and homogeneity criterion for region growing have to be supplied by the user. The former poses no problems as it can be expected that the user possesses sufficient anatomical knowledge to pinpoint a structure that he/she wants to segment. Specifying homogeneity, however, it is difficult because the user's concept of homogeneity is often vague and fuzzy and it is not translated easily into a computable criterion. Thus research in region- based segmentation has focused on the design of the growing criteria as well as on algorithm efficiency [20]. Methods can be categorized into:

DOI : $10.5121 /$ ijcsit.2012.4306 
- Criterion selection based on gray-level properties of the current points $[3,11,7]$. These methods are dependent on seed point location and search order.

- Comparison of segmentations with different homogeneity criterion $[10,18,8]$. Methods are often slow because of the large number of segmentations and they require distinguishing the true result from segmentations with slightly different homogeneity criteria.

- Criterion selection for a complete segmentation of the scene with potentially varying criterion for different regions [1,9]. The complete image has to be segmented being based on a notion of overall optimality.

We designed a new process of region growing for segmenting single structures that overcomes the limitations listed above. The process estimates the homogeneity criterion from the image itself, which produces results that are far less sensitive to the seed point selection, and allows a segmentation of individual structures. The performance of our method is compared with the adaptive moving mean value region growing method [11] because we found it is the most similar method.

\section{The Method For Automatic SeEd Selection}

\subsection{Seed Selection Criteria}

Selection of seed is based on the nature of the problem. The edge based method is used by some researchers to select seeds because the conventional seed selection method is not automatic. If the seed selection is from the center of the region segmentation becomes more effective and efficient. The three criteria for automatic seed selection are

1. Seed pixel must have high similarity to its neighbor.

2. For an expected region at least one seed must be generated in order to produce this region.

3. Seeds for different regions must be disconnected [20].

\subsection{Seeds Selecting Based on Harris Corner Detector}

Automated seed selection is the efficient time consuming criteria, we use Harris corner detect theory to realize the seed selection. The strong invariance to rotation, scale, illumination variation and image noise makes Harris corner detector a very popular detector method.

In Harris corner detector method the local autocorrelation function measures the local changes of the signal with patches shifted and the discreteness refers to the shifting of the patches [16].

Given a shift $(\delta x, \delta y)$ and a point $(x, y)$, the auto correlation function is defined as

$$
S(x, y)=\sum_{x_{i} y_{i}} W\left(x_{i}, y_{i}\right)\left[I\left(x_{i}+\delta x, y_{i}+\delta y\right)-I\left(x_{i}, y_{i}\right)\right]^{2}
$$

Where $I(x, y)$ denotes the image function and $\left(x_{i}, y_{i}\right)$ are the points in the Gaussian window $w$ centered on $(x, y)$. The window function $W$ centered on $(x, y)$ is given in $E q$. (2)

$$
W(x, y)=\frac{1}{2 \pi \sigma^{2}} e^{-\left(x^{2}+y^{2}\right) / 2 \sigma^{2}}
$$


The shifted image is approximated by a Taylor expansion truncated to the first order terms is given below in (3),

$$
I\left(x_{i}+\delta x, y_{i}+\delta y\right)=I\left(x_{i}, y_{i}\right)+\left[I_{x} I_{y}\right]\left[\frac{\delta x}{\delta y}\right]
$$

where $I_{x}$ and $I_{y}$ denote the partial derivatives in $x$ and $y$, respectively. Substituting Eq. 3 into Eq. 1, will gives

$$
S(x, y)=(\delta x, \delta y) S(x, y)\left[\frac{\delta x}{\delta y}\right]
$$

where matrix $s(x, y)$ captures the intensity structure of the local neighborhood.

Let $\lambda_{1}, \lambda_{2}$ be the eigen values of matrix $S(x, y)$.The three cases considered by analyzing the eigen vales we get first if the $\lambda_{1}$ and $\lambda_{2}$ are small, so that the local auto-correlation function is flat, that means there is little change in $S(x, y)$ in any direction, the windowed image region is of approximately constant intensity. Second, if one eigen value is high and another is low, so the local auto-correlation function is ridge shaped, then only local shifts in one direction cause little change in $S(x, y)$ and significant change in the orthogonal direction and this indicates an edge. Finally, if both eigen values are high, so the local auto-correlation function is sharply peaked, then shifts in any direction will result in a significant increase and this indicates a corner. Based on the above analysis, we use it to find the point in the uniform area. In this process, the Gaussian window size is $5 * 5, s=0.8$, and to get the most flat point and not too many points be detected we used a non minimum inhibition window, the non-minimum inhibition window size is used, the lesser seeds are selected. The threshold of $\lambda_{1}$ and $\lambda_{2}$ is also influenced the number of selected seeds. The lower threshold will generate lesser seeds and bigger region of interest. Here, the threshold is 0.01 .

Figures 1 showed the original image and the auto-selected seeds based on Harris corner detector theory. From the figures, we can see the seeds are almost felled in the homogeneous area, the seeds are satisfied the automatic seed selection criteria. The seeds are distributed evenly in the image and the different areas.

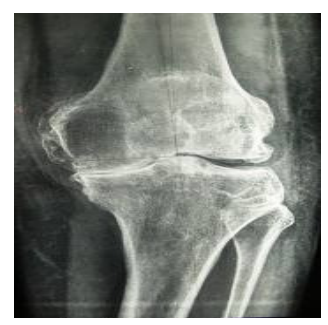

(a)

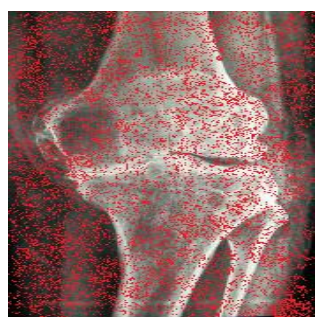

(b)

Figure 1. (a) Original image (b) Auto-selected seeds based on Harris in all image part

\section{Cloud Model Computing}

Region growing approaches exploit the important fact that pixels which are close together have similar gray values. Usually, the homogeneity predicate can be based on any characteristic of the regions in the image such as average intensity, variance, color, texture, motion, shape, size and so on. In this paper, we induced a new theory called Cloud Model theory to select segment threshold automatically, which considered the homogeneity and uncertainty of the region. 


\subsection{Cloud Model concepts}

Uncertainty is widely existed in the subjective and objective world. In all kinds of uncertainty, randomness and fuzziness are the most important and fundamental. Cloud model is an effective tool of uncertain transition between qualitative concepts and their quantitative expressions, it can express the relationship between randomness and fuzziness. It is in accord with the process of human thinking. It is a simple and effective way to simulate the uncertainty by means of knowledge representation which provides a basis for the automation of both logic and image thinking with uncertainty [11].

We suppose that $U$ is a quantitative domain represented by accurate numerical value, $U=\{x\}$ and $C$ is a qualitative concept under $U$.

If the element $x \in U$, and $x$ is a random implement of $C$, the certainty degree of $x$ to $C$ $\mu(x) \in[0,1]$ is a random number with stable tendency [12]. That is,

$$
\mu: U \rightarrow[0,1] \forall x \in U x \rightarrow \mu(x)
$$

Then, the distribution of $x$ in $U$ is called Cloud, and each $x$ is called a cloud drop. Cloud model has three numerical characteristics such as Expected value (Ex), Entropy (En), and HyperEntropy $(\mathrm{He})$, which are used to reflect the features of the concept [11]. They are very important and useful for understanding the connotation and extension of the qualitative concept.

These numerical characteristics represent quantities characteristics of an uncertainty concept. The following Figure 2 shows the cloud model with $E x=20, E n=3, H e=0.1, n=1000$ generated by Forward Normal Cloud Generator (FNCG).

$E x$ is the central value of the concept in the domain and it is the value which can most effectively represent the qualitative concept. Those elements with $E x$ value are fully compatible with the qualitative concept. In other words, the element $E x$ in the universe of discourse fully belongs to the object represented by the cloud model.

$E n$ is a measure of the concept uncertainty and it is decided by the fuzziness and randomness of the concept. On the one hand, En is a measure of qualitative concept's randomness, reflecting the dispersion degree of cloud droplet which on behalf of the qualitative concept.

On the other hand, it is also a measure of qualitative concept may be of others, reflecting the value of the cloud droplet can be taken over in the domain of the concept of space. That is, it represents the margin of the qualitative concept's double-sided property. The bigger entropy, the larger numerical scale is accepted by the fuzzier.

$H e$ is a measure of entropy uncertainty, which means that it is the entropy of entropy $(E n)$, and it reflects the discrete degree of cloud drops. It is decided by the fuzziness and randomness of En. The bigger $\mathrm{He}$ is the larger discrete degree of cloud drops which is more random. 


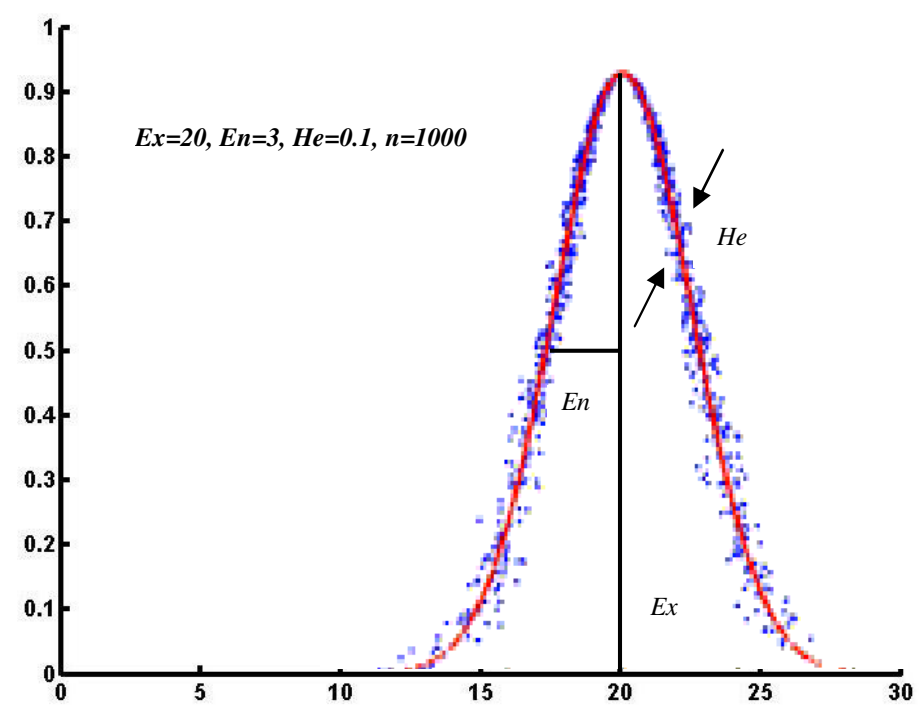

Figure 2. Cloud Model generated by FNCG

Cloud Generator $(\mathrm{CG})$ is a special computer realized algorithm. It has two kinds of generators, which are Forward Cloud Generator (CG) and Backward Cloud Generator $\left(\mathrm{CG}^{-1}\right)$. We can easily understand that the $\mathrm{CG}$ is the mapping of qualitative to quantitative, while the $\mathrm{CG}^{-1}$ the mapping of quantitative to qualitative. The $\mathrm{CG}^{-1}$ can translate some accurate data to qualitative concept which using the three numerical characteristics $(E x, E n, H e)$ to express. Homogeneity predicate is a key step in region growing segmentation procedure in which the region growing method homogeneity is an important property, which can be based on gray-level, shape, model, etc. Homogeneity criteria are based on some threshold value, the choice of which can be problematic and the segment threshold greatly affected the segment result.

We usually need to play around in order to find the right choice of thresholds, and thresholds always depend on the image data. In this paper, we use Cloud Model theory to determine the threshold of different region according to the qualitative concepts which extracted from the region around the seeds.

From the point of view of cognitive science, concept is the basic cognitive element. Concept is corresponding to a quantitative data space, and is the nature form of thinking about the object formed in the minds of human. In order to make use of the abstract concept to observe and analyze region, we must in some way to express the region into concepts. From the Cloud Model theory, we know that it's a concept express model. It reflects the homogeneity, fuzziness and randomness of a region. So, we use $\mathrm{CG}^{-1}$ to extract the concept of the region around the seed, it can get the concept's connotation and extension, which is used here as the segment threshold. Based on the analysis, image segmentation procedure and the proposed threshold auto select algorithm are described as follows.

1. Use the above Harris inner point detector of a region to get growing seeds.

2. Scan the image to get the seed $p(x, y)$, take the $p(x, y)$ as the center of a sample window, whose size is $w * w$.

3. Use $\mathrm{CG}^{-1}$ to extract the qualitative concept of the selected window, which is used to calculate the Ex, En and $\mathrm{He}$ of this window.

The three values can well express the area's character. 
International Journal of Computer Science \& Information Technology (IJCSIT) Vol 4, No 3, June 2012

4. Check the neighboring pixels and add them to the region if they are similar to the seed. Because of Ex value is fully belongs to the region (concept) represented by the cloud model.

And $E n$ is a measure of the concept uncertainty. So, take the $E x$ value as the gray center, and $E n$ as the threshold.

That is, if the neighboring pixel's gray value is between $(E x-E n, E x+E n)$, then add this pixel to the region.

5. Repeat step 4 for each of the newly added pixels; stop if there is no more pixels can be added.

Repeat step 2 to 4 until all the seeds which are not added to a region be grown.

\section{Hybrid Region Growing Algorithm}

Hybrid region growing take the medical image as the input and compute the seeds using Harris method. For every seeds it identifies a sample window of size $\mathrm{w}^{*} \mathrm{w}$ and its origin found. In the cloud model the seed regions are grown. Using canny edge detector the dominant edges of all seeds are found. Canny edge detector is the most powerful edge detector provided by the function edge. Generally edge function does not compute edges at $\pm 45^{\circ}$. To overcome this issue we need to apply the mask by use of a filtering. Here we employed the canny edge operator to find the edge correctly. The hybrid region growing algorithm is described in the flow diagram shown in Figure 3. 
International Journal of Computer Science \& Information Technology (IJCSIT) Vol 4, No 3, June 2012

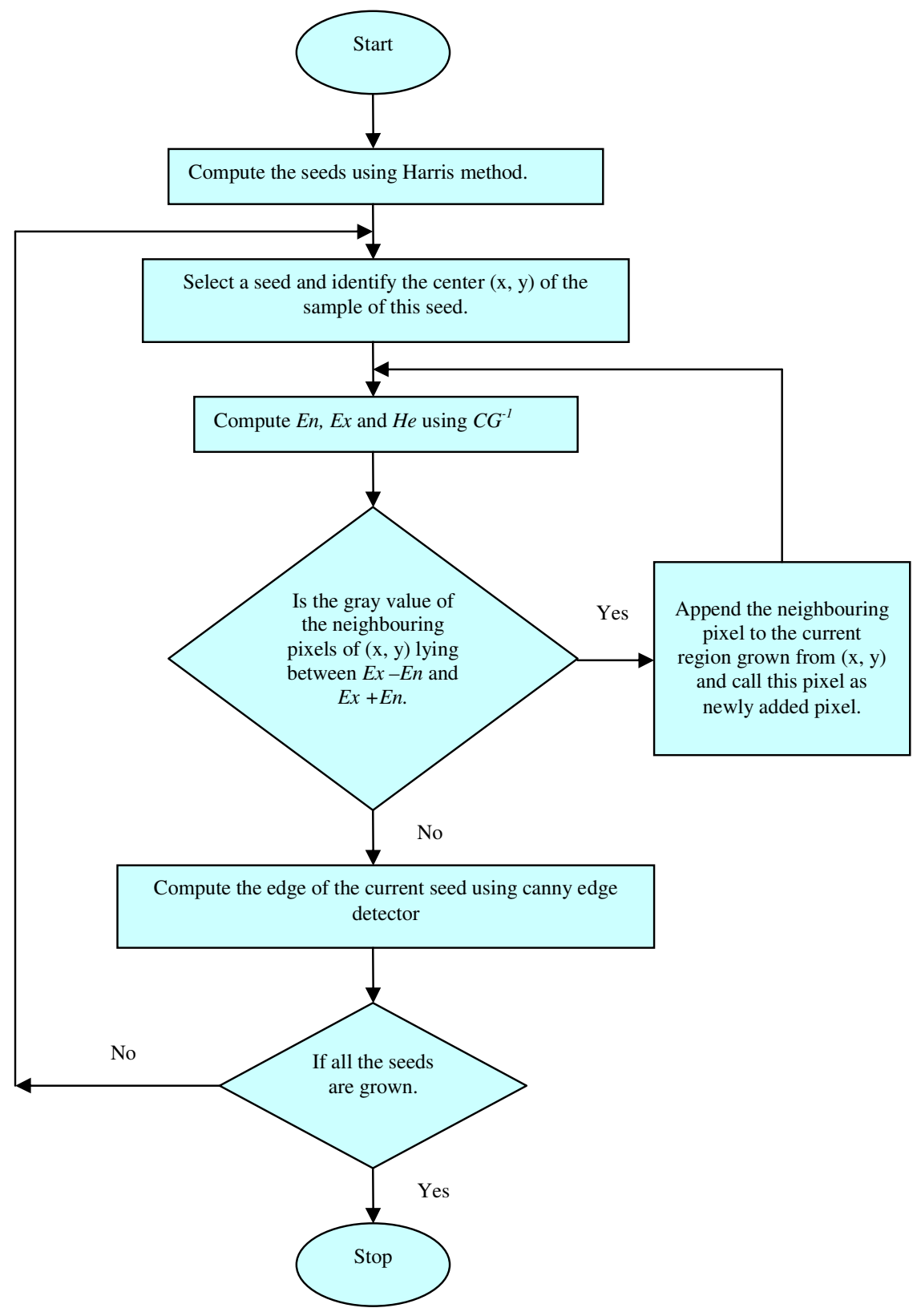

Figure 3. Flow Diagram for Hybrid region growing algorithm.

\section{RESULTS AND DISCUSSION}

To compare and evaluate the segmented result, we do some experiments on X-rays, CT scan, MR images. Use the same seeds selecting method which we expressed before and different region growing thresholds to compare and analyze the proposed segment algorithm. The segment results are showed in Figures 4 and 5. Figure 6 and 7 explains the PSNR values of segmented image with proposed edge based region growing algorithm based on maximum distance between seed pixels and minimum distance between seed pixels. 


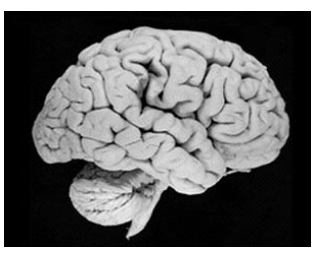

(a)

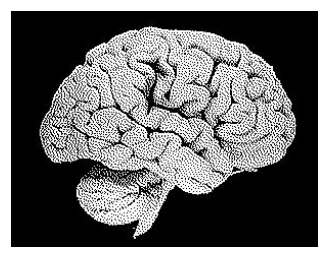

(b)

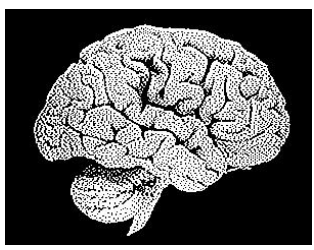

(c)

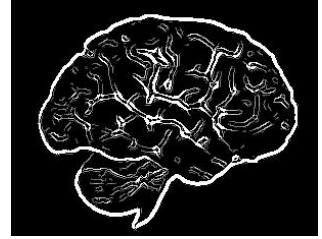

(d)

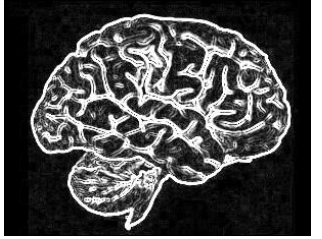

(e)

Figure 4. MRI Brain Image

(a) Original image, (b) Seed selection with maximum distance b/w seed pixels only on object,

(c) Seed selection with minimum distance b/w seed pixels only on object,

(d)segmented image with threshold, (e) Segmented image with edge based region growing

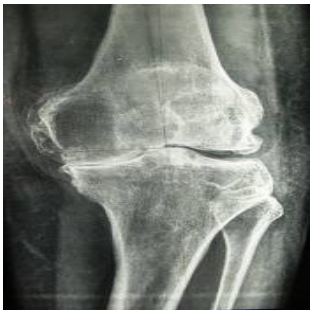

(a)

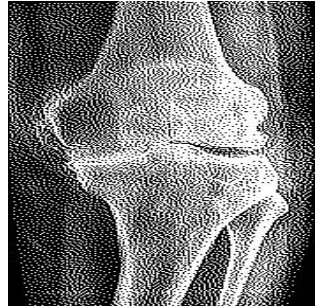

(b)

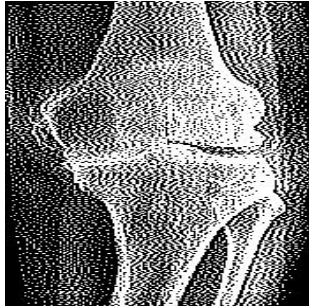

(c)

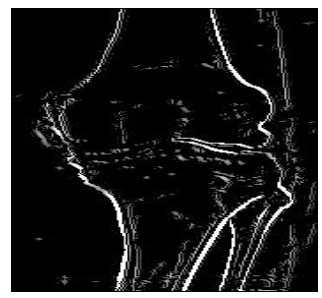

(d)

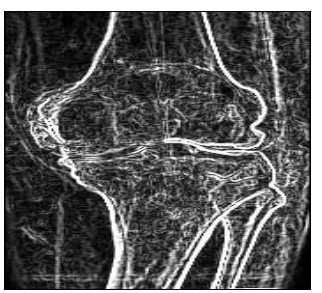

(e)

Figure 5. Bone X-ray Image

(a) Original image, (b) Seed selection with maximum distance b/w seed pixels only on object,

(c) Seed selection with minimum distance b/w seed pixels only on object,

(d)segmented image with threshold, (e) Segmented image with edge based region growing

The Following Table 1 shows the comparative picture of limitations on edge detection techniques with the proposed Hybrid Region Growing Algorithm. 
International Journal of Computer Science \& Information Technology (IJCSIT) Vol 4, No 3, June 2012

Table 1. Comparison of Edge Detection Algorithms

\begin{tabular}{|c|c|c|}
\hline $\begin{array}{l}\text { Segmentation by } \\
\text { Edge Detection }\end{array}$ & Techniques & Limitations \\
\hline Canny $[2,14]$ & Finite impulse response (FIR) filter. & $\begin{array}{l}\text { Generic threshold is difficult that works well on } \\
\text { all images. Effectiveness of the algorithm is } \\
\text { poor because of slow computation time. }\end{array}$ \\
\hline $\begin{array}{c}\text { Laplacian of } \\
\text { Gaussian } \\
{[21,13]} \\
\end{array}$ & $\begin{array}{l}\text { Difference of two multivariate } \\
\text { normal distribution. }\end{array}$ & $\begin{array}{l}\text { An inherent reduction in overall image contrast } \\
\text { produced by the operation. }\end{array}$ \\
\hline Prewitt [14] & $\begin{array}{l}\text { Template matching, convolution } \\
\text { kernels and orientation based } \\
\text { operation. }\end{array}$ & $\begin{array}{c}\text { Direct orientation estimates are not much more } \\
\text { accurate. }\end{array}$ \\
\hline Roberts [4] & Differential operation. & $\begin{array}{c}\text { Background should contribute as little noise as } \\
\text { possible. Sensitivity to noise. }\end{array}$ \\
\hline Sobel $[15,5]$ & $\begin{array}{l}\text { Discrete differentiation } \\
\text { Operation. }\end{array}$ & High frequency noise effect is not controlled. \\
\hline Zero-cross [17] & Gradient based method. & Choosing the threshold is a tough task. \\
\hline $\begin{array}{l}\text { Proposed Hybrid } \\
\text { Region Growing } \\
\text { Algorithm }\end{array}$ & $\begin{array}{l}\text { Harris corner detector based on } \\
\text { cloud model for region growing. }\end{array}$ & $\begin{array}{l}\text { It's a hybrid method will better than the } \\
\text { others. }\end{array}$ \\
\hline
\end{tabular}

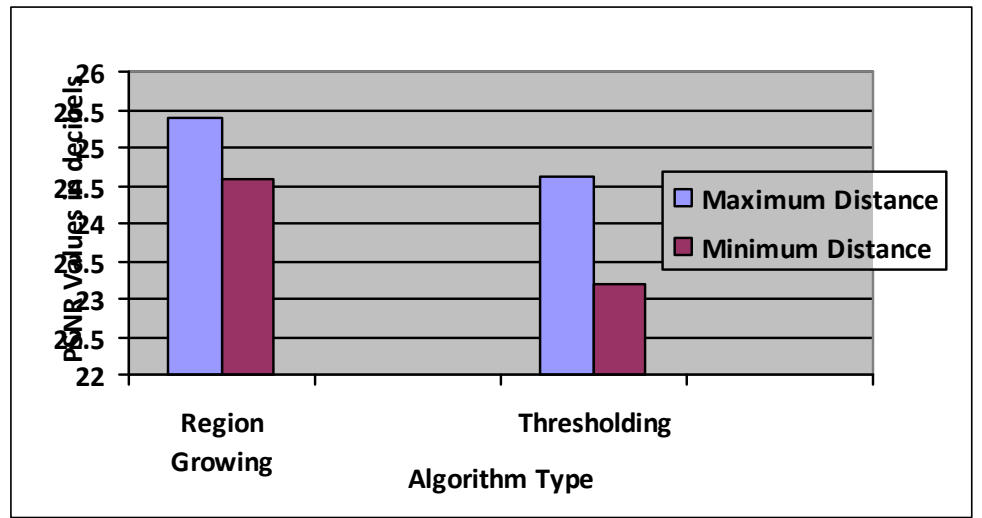

Figure 6. The PSNR values of segmented image with proposed edge based region growing algorithm for brain image with seed pixels only on objects and thresholding algorithm.

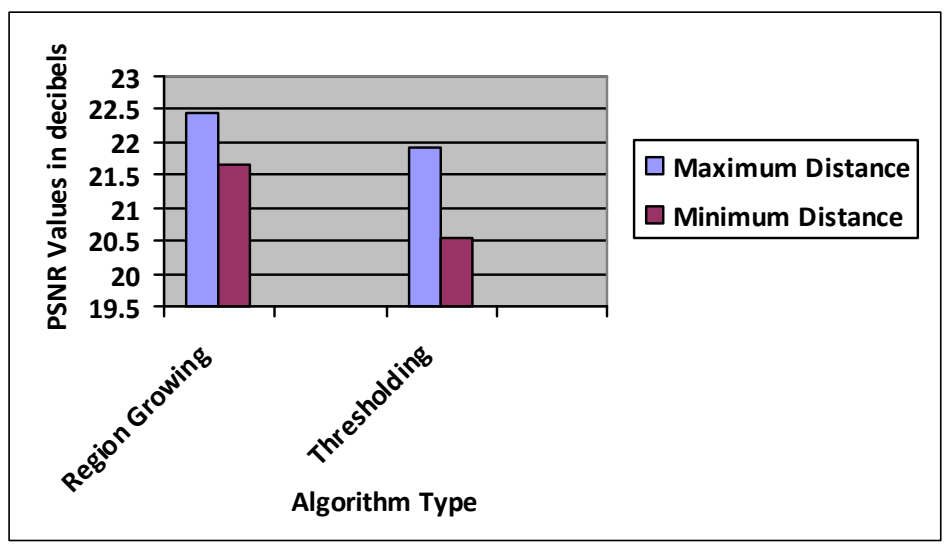

Figure 7. The PSNR values of segmented image with proposed edge based region growing algorithm for bone X-ray image with seed pixels only on objects and thresholding algorithm 


\section{CONCLuSiON}

The segment results are showed in Figures 4 and 5. The results are quite attractive. However PSNR values have been computed for quantitative performance analysis. PSNR values are as expected. The proposed hybrid region growing technique can be extended to tackle the situation where in the image boundaries are not prominent. This can address the tasks of the edges accurately even when they are blunt.

\section{REFERENCES}

[1] Adams. R \& Bischof, L., (1994) “Seeded Region Growing”, IEEE - PAMI, Vol. 16, No. 6, pp. 641- 647.

[2] Canny, J. F. (1986) "A computational approach to edge detection", IEEE Transaction on Pattern Analysis and Machine Intelligence, Vol. 8, No.6, pp. 679-697.

[3] Chang, Y.L \& Li, X., (1994) "Adaptive Image Region - Growing", IEEE Transaction on Image Processing, Vol. 3, No. 6, pp. 868-872.

[4] Davis, LS. (1975) "A survey of edge detection techniques", Computer Graphics and Image Processing, Vol. 4, No.3, pp. 248-260.

[5] Farid, H. \& E. P. Simoncelli, (2004) "Differentiation of discrete multi-dimensional signals", IEEE Transaction on Image Processing, Vol. 13, No.4, pp. 496-508.

[6] Gonzales, R.C, Woods, R.E., (1993) Digital Image Processing, Addison Wesley, Reading.

[7] Haralick, R.M \& Shapiro, L.G., (1985) "Image Segmentation Techniques", CVGIP, Vol. 29, No. 1, pp. 100-132.

[8] Hojjattolesami, S.A \& Kittler, J., (1995) "Region Growing - A New Approach", VSSP - TR, Vol. 6.

[9] Kocher, M \& Leonardi, R., (1986) "Adaptive Region Growing Techniques using Polynomial Functions for Image Approximation”, Signal Processing, No. 11, pp. 47-60.

[10] Law, T.Y \& Heng, P.A., (2000) "Automated extraction of bronchus from 3D CT images of lung based on genetic algorithm and 3D region growing”, Proc. SPIE, Vol. 3979, pp. 906-916.

[11] Levine, M \& Shaheen, M., (1981) "A Modular Computer Vision System for Image segmentations", IEEE PAMI, Vol. 3, No. 5, pp. 540-554.

[12] Li Deyi, Liu \& Changyu, (2004) "Study on the Universality of the Normal Cloud Model", Engineering Science, No. 6, pp. 28-34.

[13] Lowe, D. G. (2004) "Distinctive Image Features from Scale-Invariant Keypoints", International Journal of Computer Vision, Vol. 60, No. 2, pp. 91-110.

[14] Mamta Juneja \& Parvinder Singh Sandhu, (2009) "Performance Evaluation of Edge Detection Techniques for Images in Spatial Domain", International Journal of Computer Theory and Engineering, Vol. 1, No.5, pp. 1793-1801.

[15] Mikolajczyk, K. \& C. Schmid, (2004) "Scale and affine invariant interest point detectors", International Journal of Computer Vision, Vol. 60, No. 1, pp. 63-86.

[16] Moravec, H., (1980) "Obstacle avoidance and navigation in the real world by a seeing robot rover", Technical Report CMU-RI-TR-3, Carnegie-Mellon University, Robotics Institute.

[17] Rajiv Mehrotra \& Shiming Zhan, (1996) "A Computational Approach to Zero-Crossing-Based TwoDimensional Edge Detection”, Graphical Models and Image Processing, Vol. 58, No.1, pp. 1-17.

[18] Siebert, A., (1997) "Dynamic Region Growing”, Vision Interface '97, Kelowna.

[19] Sonka, M \& Fitzpatrick, J.M., (2000) Handbook of Medical Imaging, Vol. 2, SPIE Press.

[20] Wan, S.Y \& Higgins, W.E., (2000) "Symmetric Region Growing”, ICIP - 2000, Vancouver.

[21] Young \& Richard, (1987) "The Gaussian derivative model for spatial vision", I. Retinal mechanisms, Spatial Vision, Vol. 21, No.2, pp. 273-293 